\title{
Primeiro registro de Trichopria anastrephae, parasitoide de moscas-das-frutas, no Rio Grande do Sul
}

\author{
First record of Trichopria anastrephae, parasitoid of fruit flies, in the Rio Grande do Sul State, Brazil
}

\author{
Patrícia Postalli Cruz ${ }^{\mathrm{I}}$ Alexandre Schneid Neutzling ${ }^{\mathrm{I}}$ Flávio Roberto Mello Garcia ${ }^{\mathrm{I}^{*}}$
}

\section{- NOTA -}

\section{RESUMO}

\begin{abstract}
Existem poucas informações sobre as espécies nativas de himenópteros parasitoides de moscas-das-frutas da Região Neotropical, o que objetivou a realização do presente trabalho. Assim, coletas de goiaba (Psidium guajava L.) foram realizadas de janeiro a março de 2009 na Área Experimental em Estudos de Produção Agroecológica (AEEPA) da Universidade Federal de Pelotas, no município de Capão do Leão, Rio Grande do Sul. Os frutos foram levados ao laboratório, contados, pesados e acondicionados individualmente em potes plásticos contendo areia umedecida e telado na parte superior. Semanalmente, o substrato foi peneirado, os pupários recolhidos e acondicionados em placas de Petri com papel filtro umedecido com água destilada. Os pupários foram mantidos em sala climatizada $\left(26 \pm 2{ }^{\circ} \mathrm{C}\right.$, $60 \pm 10 \%$ de umidade relativa e $12 \mathrm{~h}$ fotofase) onde foram efetuadas observações semanais para verificar a emergência de moscas elou parasitoides e posterior identificação das espécies. Trichopria anastrephae Lima (Hymenoptera: Diapriidae) foi obtida de pupários de Anastrepha fraterculus, com parasitismo de 5,8\%.
\end{abstract}

Palavras-chave: Diapriidae, controle biológico, Anastrepha fraterculus, Tephritidae

\section{ABSTRACT}

There is little information on native species of hymenopteran parasitoids of fruit flies from the Neotropical region. Collections of guava (Psidium guajava L.) were conducted from January to March 2009 at the Experimental Area of Agroecological Production studies (AEEPA), Federal University of Pelotas, Capão do Leão, Rio Grande do Sul. Fruits were taken individually to the laboratory, counted, weighed and packaged in plastic pots containing moist sand and mesh on top. Weekly substrate was sieved, the pupae collected and placed in Petri dishes with filter paper moistened with distilled water. The pupae were placed in a room $\left(26 \pm 2^{\circ} \mathrm{C}\right.$, $60 \pm 10 \%$ RH and $12 \mathrm{~h}$ photophase) where observations were made weekly to check the emergence of adult flies or parasitoids and subsequent species identification. Trichopria anastrephae Lima was obtained from Anastrepha fraterculus in guavas, with a parasitism rate of $5.8 \%$.

Key words: Diapriidae, biological control, Anastrepha fraterculus, Tephritidae.

Existem poucas informações sobre as espécies nativas de himenópteros parasitoides de moscas-das-frutas da Região Neotropical (OVRUSKI et al., 2000). No Brasil, os principais inimigos naturais das moscas-das-frutas pertencem às famílias Braconidae, Figitidae e Pteromalidae (Hymenoptera). Pteromalidae, principalmente Pachycrepoideus vindemiae Rondani, são coletados de forma esporádica e espécimes de Figitidae são obtidos em pequenas quantidades em todo o país. Normalmente, os parasitoides coletados com maior frequência no Brasil são Braconidae (CANAL \& ZUCCHI, 2000).

A família Diapriidae, uma das maiores famílias de Hymenoptera, pertence à superfamília Proctotrupoidea e é dividida em quatro subfamílias: Belytinae, Ismarinae, Ambositrinae e Diapriinae. A família contém cerca de 150 gêneros e, aproximadamente, 2.300 espécies descritas, sendo a

'Departamento de Zoologia e Genética, Laboratório de Ecologia de Insetos, Instituto de Biologia, Universidade Federal de Pelotas (UFPel), CP 354, 96010-900, Pelotas, RS, Brasil. E-mail: flavio.garcia @pq.cnpq.br. *Autor para correspondência. 
fauna estimada em 4.500 espécies em todo o mundo (GOULET \& HUBER, 1993). O gênero Trichopria inclui microhimenópteros que podem ser utilizados para o controle biológico de moscas, visto que são parasitoides de estágios imaturos de Diptera. Trichopria anastrephae Lima é uma espécie generalista, ocorrendo geralmente um único parasitoide por pupário do hospedeiro (IORIATTI, 1995).

LIMA (1940) descreveu a espécie $\boldsymbol{T}$. anastrephae, obtida a partir de pupários de Anastrepha serpentina (Wiedemann) e Anastrepha sp. (Diptera: Tephritidae) oriundos no Rio de Janeiro. Atualmente, está distribuída nos Estados do Rio de Janeiro (LIMA, 1940; DE SANTIS 1980; AGUIAR-MENEZES et al., 2001), de Minas Gerais (SILVA et.al., 2003), de Goiás (MARCHIORI \& PENTEADO-DIAS, 2001) e de Santa Catarina (GARCIA\& CORSEUIL, 2004). Além disso, já foi registrada na Argentina (OVRUSKI et al., 2000, 2004) e na Venezuela (BOSCÁN \& GODOY, 1996). Neste trabalho, registra-se, pela primeira vez, a ocorrência de T. anastrephae para o Estado do Rio Grande do Sul.

O estudo foi realizado na Área Experimental em Estudos de Produção Agroecológica (AEEPA) localizada no campus da Universidade Federal de Pelotas (UFPel) (31 $1^{\circ} 48^{\prime} 13,8^{\prime \prime}$ S e $\left.52^{\circ} 25^{\prime} 11,7^{\prime \prime} \mathrm{W}\right)$, município de Capão do Leão, Estado do Rio Grande do Sul, Brasil. Coletas de goiaba (Psidium guajava L.) foram realizadas de janeiro a março de 2009, em árvores isoladas na área experimental. Os frutos foram levados para Laboratório de Ecologia de Insetos, contados, pesados e acondicionados individualmente em potes de plástico contendo areia umedecida e telados na parte superior. Semanalmente, o substrato foi peneirado, os pupários recolhidos e acondicionados em placas de Petri com papel filtro umedecido com água destilada. Esse processo foi repetido três vezes para cada amostra. Os pupários foram mantidos em sala climatizada $\left(26 \pm 2^{\circ} \mathrm{C}, 60 \pm 10 \%\right.$ de umidade relativa e $12 \mathrm{~h}$ fotofase $)$ onde foram efetuadas observações semanais para verificar a emergência das moscas e/ou parasitoides.
A identificação das espécies de parasitoides foi realizada baseando-se em LEGNER et al. (1976).

A relação entre uma espécie de mosca e de parasitoides foi determinada apenas quando uma única espécie de mosca foi obtida em um recipiente de emergência (CANAL et al., 1994). Foi calculada a porcentagem de parasitismo segundo SILVEIRANETO et al. (1976).

Ao total, foram coletados 54 frutos de goiaba, sendo que $89 \%$ estavam infestados por larvas de moscas-das-frutas, com um índice de infestação de $7,76( \pm 5,03)$ pupários/fruto. A viabilidade pupal foi de $55,30 \%( \pm 34,79)$. Foram obtidos 189 espécimes de Anastrepha, todos da espécie A. fraterculus (Wiedemann). Dos pupários obtidos, emergiram 52 espécimes de parasitoides, sendo eles Doryctobracon areolatus (Szepligeti) (Braconidae), Opius bellus Gahan (Braconidae), Aganaspis pelleranoi (Bréthes) (Figitidae) e T. anastrephae (Tabela 1).

Trichopria anastrephae, neste trabalho, foi obtida de pupas de Anastrepha fraterculus em goiaba, mas já havia sido constatada também em cajá-manga (Spondias dulcis Forst F.) por LIMA (1940), em carambola (Averrhoa carambola L.) por SILVA et al. (2003) e em cajá (S. mombin L.) por AGUIARMENEZES et al. (2001). Também em goiaba, porém no Estado de Santa Catarina, foi registrada por GARCIA \& CORSEUIL (2004). DE SANTIS (1980) catalogou 11 espécies de Trichopria para o Brasil e constatou que apenas T. anastrephae parasitava moscas do gênero Anastrepha.

No Brasil, são poucos os trabalhos publicados sobre a distribuição geográfica de parasitoides. Dessa forma, esses resultados aumentam o conhecimento da distribuição de T. anastrephae.

\section{AGRADECIMENTOS}

À Fundação de Amparo á Pesquisa do Estado do Rio Grande do Sul (FAPERGS), pela Bolsa de Iniciação Científica

Tabela 1 - Parasitoides de Anastrepha fraterculus, com respectivo número de espécimes e porcentagem de parasitismo, obtidos de frutos de goiaba na Área Experimental em Estudos de Produção Agroecológica (AEEPA), Capão do Leão, Rio Grande do Sul. Janeiro a março de 2009.

\begin{tabular}{lccc}
\hline Parasitoides & Número espécimes & Porcentagem parasitismo (\%) & Frequência (\%) \\
\hline $\begin{array}{l}\text { Braconidae } \\
\begin{array}{l}\text { Doryctobracon areolatus } \\
\text { Opius bellus }\end{array}\end{array}$ Figitidae $^{\text {Aganaspis pelleranoi }}$ & 17 & 24,9 & 90,4 \\
$\begin{array}{l}\text { Diapriidae } \\
\text { Trichopria anastrephae }\end{array}$ & 1 & 0,5 & 1,9 \\
\hline
\end{tabular}


do segundo autor e ao Conselho Nacional de Desenvolvimento Científico e Tecnológico $(\mathrm{CNPq})$, pela Bolsa de Produtividade em Pesquisa do terceiro autor.

\section{REFERÊNCIAS}

AGUIAR-MENEZES, E.L. et al. Native hymenopterous parasitoids associated with Anastrepha spp. (Diptera: Tephritidae) in Seropedica city, Rio de Janeiro, Brazil. Florida Entomologist, v.84, n.4, p.706-711, 2001. Disponível em: <http://www.fcla.edu/ FlaEnt/fe84p706.pdf>. Acesso em: 27. fev. 2001.

BOSCÁN, N. DE M; GODOY, F. Nuevos parasitoides de moscas de las frutas de los generos Anastrepha Y Ceratitis en Venezuela. Agronomía Tropical, v.4, n.4, p.465-471, 1996.

CANAL, N.A. et al. Reconocimiento de las especies de parasitoides (Hym.: Braconidae) de moscas de las frutas (Dip.: Tephritidae) en dos municipios del Estado de Amazonas, Brasil. Boletin Museo Entomologico de La Universidad del Valle, v.2, p.1-17, 1994.

CANAL, N.A.; ZUCCHI, R.A. Parasitóides Braconidae. In MALAVASI, A.; ZUCCHI, A. Moscas-das-frutas de importância econômica no Brasil, Conhecimento básico e aplicado. Ribeirão Preto: Holos, 2000. p.119-126.

DE SANTIS, L. Catalogo de Los himenopteros brasileños de la Serie Parasitica; Incluyendo Berthyloidea. Curitiba: Universidade Federal do Paraná. 1980. 395p.

GARCIA, F.R.M.; CORSEUIL, E. Native hymenopteran parasitoids associated with fruit flies (Diptera: Tephritoidea) in Santa Catarina State, Brazil. Florida Entomologist, v.87, n.4, p.517-521, 2004. Disponível em: <http:// www.bioone.org/doi/abs/10.1653/00154040\%282004\%29087\%5B0517\%3ANHPAWF\%5D2.0.CO\%3B2>. Acesso em: 28 set. 2011. doi: 10.1653/00154040(2004)087 [0517:NHPAWF]2.0.CO;2

GOULET, H.; HUBER, J.T. Hymenoptera of the world: an identification guide to families. Toronto: Agriculture Canada Puclications, 1993. 668p.

IORIATTI, M.C.S.S. Contribuição ao estudo da biologia e taxonomia de Hymenoptera parasitóides de Diptera das famílias Tephritidae e Lonchaeidade. 1995. 124f.
Dissertação (Mestrado em Ecologia) - Universidade Federal de São Carlos, SP.

LEGNER, E.E. et al. Tabular keys \& biological notes to commom parasitoids of synanthropic Diptera breeding in accumulated animal wastes. Entomological News, Philadelphia, v.87, p.113$144,1976$.

LIMA, A.C. Alguns parasitos de moscas de frutas. Anais da Academia Brasileira de Ciência, v.12, n.1, p.17-20, 1940.

MARCHIORI, C.H.; PENTEADO-DIAS, A.M. Trichopria anastrephae (Hymenoptera: Diapriidae), parasitóide de Diptera, coletadas em área de mata nativa e pastagem em Itumbiara, Goiás, Brasil. Arquivos do Instituto Biológico, v.68, n.1, p.123-124, 2001. Disponivel em: <http://www.biologico.sp.gov.br/docs/arq/ V68_1/23.pdf>. Acesso em: 28 fev. 2011.

OVRUSKI, S.M. et al. Hymenopteran parasitoids on fruitinfesting Tephritidae (Diptera) in Latin America and the southern United States: Diversity, distribution,taxonomic status and their use in fruit fly biological control. Integrated Pest Management Reviews, v.5, p.81-107, 2000. Disponível em: <http://www.springerlink.com/content/pr8382w1035lk170/>. Acesso em: 28 fev. 2011. doi: 10.1023/A:1009652431251.

OVRUSKI, S.M. et al. Indigenous parasitoids (Hymenoptera) attacking Anastrepha fraterculus and Ceratitis capitata (Diptera: Tephritidae) in native and exotic host plants in Northwestern Argentina. Biological Control, v.29, p.4357, 2004. Disponível em: <http://www.sciencedirect.com/ science?_ob=ArticleURL\&_udi=B 6WBP-4938JF7$1 \&$ _user $=10 \&$ \&coverDate $=01 \% 2 \mathrm{~F} 31 \% 2 \mathrm{~F} 2004 \&$ rdoc $=1 \&$ fm $\mathrm{t}=\mathrm{high} \&$ orig=gateway\&_origin=gateway\&_sort $=\mathrm{d} \&$ _docanchor $=\& v i e w=c \& \_s e a r c h S t r I d=1659307556 \&$ rerunOrigin $=$ goog 1 e\&_acct $=$ C000050221\&_version $=1$ \&_urlVersion $=0$ \&_userid $=10 \&$ md5=bf154048538f1f5f9e1 ebc9ae188d69c\&searchtype $=a>$. Acesso em: 28 fev. 2011. doi:10.1016/S1049-9644(03)00127-0.

SILVA, C.G. et. al. Himenópteros parasitóides de larvas de Anastrepha spp. em frutos de carambola (Averrhoa carambola L.) na região de Divinópolis, Minas Gerais, Brasil. Ciência e Agrotecnologia, v.27, n.6, p.1264-1267, 2003. Disponível em: <http://www.scielo.br/scielo.php? pid=S 1413 $70542003000600009 \&$ script $=$ sci_arttext $>$. Acesso em: $28 \mathrm{fev}$. 2011. doi: $10.1590 / \mathrm{S} 1413-70542003000600009$.

SILVEIRA NETO, S. et al. Manual de ecologia dos insetos. Piracicaba: Agronômica Ceres, 1976. 419p. 